\title{
Autismo Cuidados Primários de Saúde
}

Guiomar Oliveira*

\section{RESUMO}

O autismo é uma perturbação complexa e crónica do neurodesenvolvimento, habitualmente grave e frequente. Pretende-se com este manuscrito, e à luz do conhecimento actual, dar orientações sobre como vigiar e rastrear os sinais patológicos que o caracterizam, os quais, na maioria dos casos, são evidentes antes dos dois anos de idade. Fazem parte da tríade semiológica específica do autismo as dificuldades nas relações sociais e na comunicação verbal e não verbal e um comportamento rígido e repetitivo. É proposta a aplicação de um teste de rastreio específico de autismo, The Modified Checklist for Autism in Toddlers (M-CHAT), aos 18 e 24 meses. Os casos suspeitos devem ser orientados para avaliação especializada multidisciplinar em Unidades ou Centros de Desenvolvimento.

Palavras-chave: Autismo; Perturbações Espectro Autismo; Sinais; Rastreio.

\section{INTRODUÇÃO}

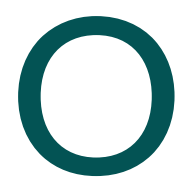

O autismo, termo utilizado neste texto como sinónimo de perturbações do espectro do autismo (PEA), refere-se a uma perturbação neurológica que, clinicamente, se manifesta por atraso ou desvio nas aquisições do neurodesenvolvimento e por alterações do comportamento que, na grande maioria dos casos, são evidentes antes dos dois anos de idade.

A disfunção cerebral que está subjacente ao autismo compromete a progressão de todo o neurodesenvolvimento, podendo o quadro clínico inicial assemelhar-se ao atraso psicomotor global. No entanto, as dificuldades de interacção e comunicação social associadas a comportamentos repetitivo permitem diferenciar o autismo de outras perturbações do neurodesenvolvimento.

Esta entidade foi pela primeira vez descrita por Leo Kanner em 1943, que então a denominou de «Autistic disturbances of affective contact». ${ }^{1}$ Quase em simultâneo, Hans Asperger, desconhecendo a publicação de Kanner, descreveu o mesmo quadro clínico mas em crianças de idade escolar, apelidando-o «Autistic psy-

*Unidade de Neurodesenvolvimento e Autismo

Centro Desenvolvimento Luís Borges, Hospital Pediátrico - Centro Hospitalar Coimbra, Portugal

Presidente da Sociedade de Pediatria do Neurodesenvolvimento da Sociedade Portuguesa de Pediatria chopathy». ${ }^{2}$ Estes autores, ao verificarem que a clínica do autismo era muito precoce e que o défice na interacção social era evidente desde os primeiros meses de vida, consideraram esta patologia inata e de causa biológica. ${ }^{1}$ Pelo contrário, a influência da Psicanálise nos anos 50 e 60 converteu o autismo numa perturbação emocional. A parentectomia (afastamento das crianças do núcleo familiar considerado disfuncional) era então um método terapêutico corrente. ${ }^{3}$ Esta conotação negativa e dolosa do autismo foi de tal maneira marcante que, ainda hoje, meio século depois, para alguns profissionais o diagnóstico de autismo deve ser ocultado da família.

Nas décadas seguintes vários estudos reforçaram a existência desta entidade clínica e delinearam critérios de diagnóstico. ${ }^{4,5}$ Provaram a sua heterogeneidade etiológica, demonstrando que a grande maioria dos casos é de causa genética com marcada hereditariedade. ${ }^{6}$ Somente em 1980, na $3^{a}$ edição do manual de diagnóstico das doenças mentais da Associação Americana de Psiquiatria (AAP), Diagnostic and Statistical Manual of MentalDisorders (DSM), é que o autismo infantil foi incluído numa nova classe de perturbações, Pervasive Developmental Disorders (PDD), termo adaptado para português como Perturbações Globais do Desenvolvimento (PGD).

Na revisão de 1987 (DSM-III-R), considerou-se como nuclear no quadro de autismo a presença de uma tría- 
de clínica: marcado défice na interacção e comunicação social em relação ao esperado para a idade mental da criança e ainda um comportamento repetitivo. ${ }^{7}$ Actualmente estão em uso os critérios de diagnóstico da AAP de 1994 (DSM-IV), com a revisão do texto em 2000 (DSM-IV-TR) que são sobreponíveis aos utilizados na Classificação Internacional de Doenças. ${ }^{8,9,10}$

Os critérios de diagnóstico de perturbação autística (autismo clássico), síndrome de Asperger (autismo ligeiro, com bom potencial linguístico e cognitivo) e perturbação global do desenvolvimento sem outra especificação (PGD/SOE) ou autismo atípico (apenas algumas características de autismo) que compõem as perturbações do espectro do autismo (PEA), estão registados nos Quadros I e II.

O autismo é frequente e em Portugal a prevalência estimada é de aproximadamente um caso em cada 1.000 crianças de idade escolar. ${ }^{11} \mathrm{O}$ diagnóstico precoce, bem como uma avaliação adequada e uma intervenção atempada e intensiva, melhoram o prognóstico. ${ }^{12}$ Embora o seguimento destas crianças e famílias deva estar a cargo de equipas multidisciplinares de Unidades e Centros de Desenvolvimento, a detecção precoce é do domínio dos cuidados primários de saúde. Actualmente está documentado um atraso de um a dois anos entre o início das queixas e o diagnóstico de autismo. ${ }^{12}$ Esta revisão pretende dar a conhecer dados clínicos e normas de vigilância e rastreio para a identifi-

\section{QUADRO I. Critérios de diagnóstico de perturbação autística (autismo clássico), segundo a DSM-IV-TR}

A. No global, presença de seis ou mais critérios na interacção social (IS), na comunicação (C) e no comportamento repetitivo (CR), pelo menos dois de IS, um de $C$ e um de $C R$.

Défice qualitativo na IS, manifestado pelo menos por duas das seguintes características:

a) acentuado défice no uso de múltiplos comportamentos não verbais, tais como o contacto visual, a expressão facial, a postura e os gestos reguladores da interacção social;

b) incapacidade para desenvolver relações com os companheiros, adequadas ao nível de desenvolvimento;

c) ausência da tendência espontânea para partilhar prazeres, interesses ou objectivos (por exemplo, não mostrar, trazer ou indicar objectos de interesse) com os outros;

d) falta de reciprocidade social ou emocional (alteração na sintonia emocional com os outros).

Défice qualitativo na $C$, manifestado pelo menos por uma das seguintes características:

a) atraso ou ausência total de desenvolvimento da linguagem oral (não acompanhada de tentativas para compensar através de modos alternativos de comunicação, tais como gestos ou mímica);

b) uma acentuada incapacidade na competência para iniciar ou manter uma conversação com os outros, nos sujeitos com um discurso adequado;

c) uso estereotipado ou repetitivo da linguagem ou linguagem idiossincrática;

d) ausência de jogo realista espontâneo, variado, ou de jogo social imitativo adequado ao nível de desenvolvimento.

Défice qualitativo no $C R$, manifestado pelo menos por uma das seguintes características:

a) preocupação absorvente por um ou mais padrões estereotipados e restritivos de interesses que são anormais, quer pela intensidade quer pelo objectivo;

b) adesão, aparentemente inflexível, a rotinas ou rituais específicos, não funcionais;

c) maneirismos motores estereotipados e repetitivos (por exemplo, sacudir ou rodar as mãos ou dedos ou movimentos complexos de todo o corpo);

d) preocupação persistente com partes de objectos.

B. Atraso ou funcionamento anormal em pelo menos uma das áreas (IS, C, CR) com início antes dos três anos de idade.

C. A perturbação não é melhor explicada pela presença de uma perturbação de Rett ou perturbação desintegrativa da segunda infância.

NOTA: Perturbação global do desenvolvimento sem outra especificação (PGD/SOE) ou autismo atípico, para as crianças que apresentam alguns critérios de perturbação autística, mas em que o número ou a gravidade não são suficientes para este diagnóstico; ou em que a sintomatologia surgiu após os três anos de idade. 


\section{QUADRO II. Critérios de diagnóstico DSM-IV-TR de perturbação de Asperger}

A. Défice qualitativo na IS, manifestado pelo menos por duas das seguintes características:

a) acentuado défice no uso de múltiplos comportamentos não verbais, tais como o contacto visual, a expressão facial, a postura e os gestos reguladores da interacção social;

b) incapacidade para desenvolver relações com os companheiros, adequadas ao nível de desenvolvimento;

c) ausência da tendência espontânea para partilhar prazeres, interesses ou objectivos (por exemplo, não mostrar, trazer ou indicar objectos de interesse) com os outros;

d) falta de reciprocidade social ou emocional (alteração na sintonia emocional com os outros).

B. Padrões de comportamento, interesses e actividades restritos, repetitivos e estereotipados, que se manifestam pelo menos por uma das seguintes características:

a) preocupação absorvente por um ou mais padrões estereotipados e restritivos de interesses que são anormais, quer pela intensidade quer pelo objectivo;

b) adesão, aparentemente inflexível, a rotinas ou rituais específicos, não funcionais;

c) maneirismos motores estereotipados e repetitivos (por exemplo, sacudir ou rodar as mãos ou dedos ou movimentos complexos de todo o corpo);

d) preocupação persistente com partes de objectos.

C. A perturbação produz um défice clinicamente significativo da actividade social, laboral ou de outras áreas importantes do funcionamento.

D. Não há um atraso global da linguagem clinicamente significativo (ou seja, deve usar palavras simples aos dois anos e frases comunicativas aos três)

E. Não há atraso clinicamente significativo no desenvolvimento cognitivo ou no desenvolvimento de aptidões de auto-ajuda próprias da idade, no comportamento adaptativo (distinto da interacção social) e na curiosidade acerca do meio ambiente durante a infância.

F. Não preenche os critérios para autismo ou esquizofrenia.

cação e orientação precoces das crianças com perturbação do espectro do autismo (PEA).

\section{VIGILÂNCIA VERSUS RASTREIO DO DESENVOLVIMENTO NOS PRIMEIROS ANOS}

Os médicos que realizam consultas de primeira linha em crianças na grande maioria saudáveis devem estar preparados para vigiar e detectar precocemente as perturbações do neurodesenvolvimento que, na sua globalidade, afectam 15 a $20 \%$ das crianças. ${ }^{13}$

A vigilância do desenvolvimento é um processo contínuo e flexível de observações qualificadas em consultas de rotina, que não implica o recurso a testes. Já o rastreio subentende a aplicação de um teste desenhado para identificar problemas específicos do desenvolvimento em idades consideradas chave..$^{14,15}$

$\mathrm{O}$ reconhecimento precoce de atrasos ou desvios do desenvolvimento requer um conhecimento prévio e bem sedimentado do modo como se avalia e interpreta a progressão das etapas do desenvolvimento e a sua variabi- lidade normal. O neurodesenvolvimento é um processo muito natural e dinâmico, com grandes diferenças individuais e que se processa por surtos (a uma velocidade não uniforme) As alterações ligeiras não são fáceis de detectar, embora sejam sempre preocupantes no sentido em que, frequentemente, têm por base uma disfunção neurológica, sensorial ou, mais raramente, ambiental. As várias áreas em que, classicamente, para melhor avaliação e compreensão, se divide o desenvolvimento psicomotor global, como a motricidade global, a visuomotricidade a linguagem (compreensão e expressão) e o comportamento adaptativo/autonomia, estão, na verdade, interligadas. A sua monitorização (baseada na avaliação de capacidades progressivamente mais complexas) deve ser realizada em todas as consultas de rotina para que se possa desenhar a sua curva evolutiva.

São consideradas idades chave de rastreio dos problemas globais do neurodesenvolvimento as consultas de rotina dos 9 , 18 e 24 ou 30 meses. $^{14}$

O autismo resulta de disfunção neurológica com iní- 
cio prénatal, como o demonstram estudos neuropatológicos que evidenciaram anomalias de desenvolvimento cerebral precocemente no feto. ${ }^{16,17}$ Apesar da precocidade neuropatológica com repercussão clínica nas aquisições do desenvolvimento, o diagnóstico de autismo acontece tardiamente, na maior parte das vezes entre os quatro e os cinco anos. ${ }^{13}$ No entanto, como seria de esperar, as preocupações relativamente ao comportamento e à evolução do desenvolvimento são bastante anteriores, surgindo habitualmente por volta dos 18 meses, essencialmente por atraso de linguagem, que é a alteração mais evidente para os cuidadores. Daí que os clínicos e as associações médicas venham envidando esforços para que os sinais precoces de autismo, prévios ao atraso de linguagem, sejam reconhecidos logo no primeiro e segundo anos de vida, alertando os médicos de cuidados primários nesse sentido, uma vez que o atraso do diagnóstico resulta essencialmente do desconhecimento semiológico do comportamento social precoce e pré-linguístico.

\section{O AUTISMO NOS PRIMEIROS DOIS ANOS DE VIDA}

Neste período, em regra, o autismo manifesta-se por atraso nas aquisições do desenvolvimento global e ausência de comportamentos normais esperados para a idade mental e não pela presença de comportamentos mais conhecidos, como as estereotipias e as fixações (abanar as mãos, rodopiar objectos, fixar-se na máquina de lavar, nos botões dos electrodomésticos, em luzes, etc.) ${ }^{18}$ Cerca de dois terços das crianças com autismo também sofrem de deficiência mental. Deste modo, será importante identificar precocemente sinais específicos, que possam distinguir o autismo de outra entidade com que se pode confundir e que é o atraso global de desenvolvimento (modo de apresentação do défice intelectual nos primeiros três anos de vida). As crianças com autismo mas sem défice intelectual, têm somente afectadas as áreas da relação e da comunicação social. ${ }^{18,19}$ Estudos de filmes de festas do primeiro aniversário concluíram que as crianças com autismo se distinguiam das outras que apenas vinham a apresentar deficiência mental por apresentarem menos interesse social (não «respondendo» ao olhar dos outros ou quando eram chamados, não olhando para as pessoas nem para os objectos que estas lhes mostravam), revelando assim grande défice na partilha de interesses so- ciais e visuais. ${ }^{19}$

A atenção conjunta («joint attention») é um sistema de processamento de informação que se inicia entre os quatro e os seis meses de idade e onde se alicerça a aprendizagem social. Baseia-se na capacidade inata de a criança coordenar o seu olhar (atenção visual) com o do seu parceiro social, o que leva a uma partilha de interesses visuais que acabam por ser modelos sociais de ensino e aprendizagem..$^{20}$

Dois tipos de comportamento de atenção conjunta podem ser observados muito claramente nos primeiros meses de vida. O primeiro, refere-se à aptidão da criança seguir o olhar e os gestos (ex: apontar) do parceiro social e, deste modo, partilhar a sua referência visual. É a capacidade de responder à atenção conjunta, ou seja, de a criança seguir «a indicação» dada pelo «interlocutor» para um objecto ou evento. O outro, refere-se à capacidade da criança iniciar a atenção conjunta, espontaneamente, ela própria, indicando por olhar ou gestos (estender a mão ou apontar) o objecto ou evento que pretende pedir ou mostrar ao parceiro social, mas certificando-se (por trocas de olhar e de expressão facial) de que o outro deu conta da sua indicação. ${ }^{20}$

Esta partilha na interacção visual/social precoce está sistematicamente afectada no autismo e a sua ausência ou défice é actualmente considerada a apresentação clínica mais precoce e exclusiva do autismo. Neste âmbito é indispensável que os clínicos dominem bem a sua progressão normal. Aos nove meses o bebé já é capaz de seguir visualmente o foco de interesse que lhe é mostrado. Ao ano de idade já olha, vocaliza ou aponta para o objecto que necessita, pedindo-o (apontar protoimperativo). Entre os 16 e os 18 meses, já mais evoluídos socialmente, apontam não só para pedir mas também para partilhar o prazer (apontar protodeclarativo).$^{19}$

A consulta de rotina dos 9 meses é, neste contexto, essencial. Nesta idade as capacidades comunicativas são bem evidentes como responder, e mesmo iniciar, a atenção conjunta. Dificuldades no contacto visual, na imitação de gestos e "gracinhas», em ligar ao nome, em vocalizar e em adquirir gestos comunicativos, são indícios fortes de autismo. ${ }^{15,18}$ Deve-se aproveitar este encontro entre a família e a equipa dos cuidados primários de saúde para alertar os pais acerca destas capacidades, da sua importância como indicadores pré-linguísticos e de toda a aprendizagem verbal e social. 
De facto, na maioria dos casos de autismo estes sinais de desinteresse social já existem, mas é só aos 18 meses que os pais dão conta que não surgem as cerca de dez palavras com significado esperadas e que não obedecem a ordens simples (ex: identificar três partes do corpo ou mais de cinco objectos de uso comum). A suspeita de défice auditivo é atenuada pela reacção clara que a criança revela aos seus estímulos sonoros de interesse. Nesta fase, deve-se então estar atento aos desvios de desenvolvimento como seja a capacidade de identificar números, letras, logótipos, matrículas de automóvel e sinais de trânsito, de entre outras, que não são de esperar numa criança que ainda não fala. É muitas vezes um desenvolvimento desarmonioso que, associado a défices comunicativos, denuncia a presença de um quadro de autismo.

Efectivamente, estudos prospectivos muito actuais têm colocado em evidência que, entre os 12 e os 18 meses, as crianças mais tarde diagnosticadas com autismo já apresentavam anomalias em uma ou mais das seguintes áreas: visual (interesse visual atípico, mais fixado nos objectos que na face humana); motora (atraso nas aquisições motoras tanto na motricidade fina como global e maneirismos motores); brincar (atraso na imitação motora, manuseio dos objectos limitado a movimentos repetitivos em vez de exploração funcional e simbólica, por exemplo, rodopiar as rodas do carro em vez de o usar para brincar); comunicação social (olhar desviante, dificuldades em responder ao nome e em imitar, pouco interesse social, pouca partilha emocional positiva); linguagem (atraso na vocalização recíproca - resposta sonora e expressiva facial e corporal do bebé à interacção do seu parceiro social - e na aquisição das palavras, bem como défice na compreensão de ordens e dos gestos); e dificuldades em aprender actividades diárias. ${ }^{21,22}$ Por outro lado, é de realçar que, paradoxalmente, estas crianças podem parecer mais autónomas que os seus pares, o que por vezes dá uma falsa segurança quanto à normalidade do desenvolvimento. Quando querem algo que não está ao seu alcance, a primeira estratégia não é pedir ao adulto (olhando, vocalizando, apontando, como é normal), é antes tentar alcançá-lo por outros meios (trepando, arrastando). Em último caso, podem mover o adulto puxando-o pela mão, com pouco ou nenhum contacto visual, como se este fosse um objecto.

\section{AUTISMO: VIGILÂNCIA, RASTREIO E ORIENTAÇÃO}

Nas consultas de rotina de saúde infantil, ou em qualquer outra suplementar agendada por suspeita do médico ou dos familiares no que concerne a preocupações sobre autismo, devem ser seguidos os preceitos considerados necessários para uma boa vigilância de desenvolvimento, que são: ouvir as preocupações dos pais acerca do desenvolvimento e comportamento da criança, colher a história clínica e de desenvolvimento, observar cuidadosamente a criança neste contexto, identificar factores de risco ou de protecção e proceder ao registo sucessivo dos dados. Desta análise, deve emergir a presença ou ausência dos factores considerados de risco para autismo: 1 - ter irmão/s com autismo, 2- os pais estarem preocupados com o desenvolvimento e/ou comportamento, 3- outras pessoas que convivem com a criança estarem preocupadas, 4 - o médico ficar preocupado (cada uma destas alíneas vale um, o risco máximo terá uma cotação de quatro).

Se não houver qualquer factor de risco, a cotação será zero. Contudo, se se tratar da consulta dos 18 ou 24 meses, apesar de baixo risco sugere-se a administração de um teste de rastreio para autismo. Se o resultado do teste de rastreio for negativo deve entrar de novo no esquema das consultas de rotina, se for positivo deve ser orientado para uma consulta especializada.

Se a cotação dos factores de risco for 1 e se a criança tiver pelo menos 18 meses, deve administrar-se um teste de rastreio para autismo. Se o resultado for positivo deve ser orientada para consulta especializada. Se for negativo, deve-se chamar a atenção dos pais para sinais de autismo e marcar outra consulta dentro de um mês para reavaliação. Se o factor de risco for ter um irmão com autismo, deve-se manter um elevado nível de suspeição, mas não é recomendada a reavaliação dentro de um mês. Se apresentar um factor de risco mas tiver menos de 18 meses, não havendo, actualmente, testes de rastreio disponíveis, deve reforçar-se a vigilância das capacidades de comunicação social e seguir o percurso proposto para a criança com 18 meses ou mais.

Se a criança tiver dois ou mais factores de risco deve imediatamente ser orientada para avaliação especializada, onde se procederá a uma avaliação formal do neurodesenvolvimento e do comportamento.

A escolha do teste de rastreio a adoptar não é fácil 
uma vez que estes testes acarretam sempre um risco elevado de falsos positivos, sobretudo aqueles que se baseiam apenas em questionários dirigidos aos pais e dos quais não fazem parte tarefas de observação directa (mas que têm, contudo, a vantagem de ser mais rápidos). No entanto, a utilização de instrumentos específicos para rastreio tem-se imposto pelo facto de apenas a impressão clínica e a vigilância, mesmo por clínicos experientes, apresentar uma sensibilidade muito baixa (20 a 30\%) para o diagnóstico de autismo. ${ }^{21}$ Assim, um teste de rastreio deve utilizar-se sempre nos casos suspeitos e, como proposto pela Academia Americana de Pediatria, em todas as crianças na consulta dos 18 meses. Para que se minimize o número de casos falsos negativos, um grupo de peritos sugere a repetição do teste de rastreio entre os 24 e os 30 meses, de modo a detectar os casos mais ligeiros ou com regressão no segundo ano de vida. ${ }^{15}$

$\mathrm{O}$ instrumento de rastreio que se propõe (The $M o$ dified Checklist for Autism in Toddlers, M-CHAT) ${ }^{21}$ foi traduzido para português com a autorização da autora Diana Robins, e está acessível online, assim como a respectiva cotação. ${ }^{22}$ É um questionário muito simples, composto por 23 perguntas (de resposta sim ou não), que deve ser preenchido pelos pais/cuidadores, não necessitando de qualquer preparação prévia. A validade desta escala tem sido bem estudada no grupo etário entre os 16 e os 30 meses. Apesar da boa sensibilidade (85\%) e especificidade (93\%), apresenta um valor preditivo positivo baixo o que, no caso de um resultado positivo, implica não prescindir da observação da criança nem do esclarecimento das respostas dadas antes da referenciação. A aplicação da escala é muito rápida (dois minutos). São considerados resultados positivos quando há falha em pelo menos 3 das 23 perguntas ou em 2 das 6 consideradas críticas. ${ }^{21,23,24}$

O diagnóstico de autismo, quando efectuado por clínicos experientes em centros de referência, é seguro mesmo se realizado precocemente; contudo, o risco de falsos positivos é sempre de considerar. No entanto, nos casos raros em que essa circunstância se vem a verificar, as crianças na verdade apresentam outras perturbações do neurodesenvolvimento, particularmente problemas do desenvolvimento da linguagem, comportamentais e défices sensoriais, que exigem também intervenção precoce. O seguimento permitirá o diag- nóstico mais preciso da patologia do neurodesenvolvimento em causa. Nos casos em que o diagnóstico de autismo é suspeito, mas em que ainda não é claro, deve-se orientar de imediato para intervenção educativa dos défices de desenvolvimento e das alterações do comportamento detectados e manter a avaliações sucessivas. Estudos indicam que os pais preferem ser informados da suspeita. A manutenção da dúvida, adiando o diagnóstico e a intervenção, é angustiante para as famílias. ${ }^{25,26}$

A intervenção no autismo é essencialmente educacional, devendo recorrer-se a estratégias comportamentais e terapias directas que devem ser do conhecimento da família. Numa fase precoce, as crianças em risco ou já com o diagnóstico de autismo devem ser intensivamente ensinadas a comunicar recorrendo a meios aumentativos e alternativos (símbolos, gestos, imagens, de preferência em contextos naturais), a adequar comportamentos sociais, a brincar, a adquirir autonomia e a controlar comportamentos disruptivos (birras, agitação, sono e alimentação). ${ }^{12}$ Devem ser então requisitados os serviços de intervenção precoce locais (decreto-lei n²81/2009 de 6 de Outubro) e as unidades de ensino estruturado para alunos com autismo. Estes núcleos devem dispor de recursos de terapia da fala de acordo com o decreto-lei no 3/2008 da lei da educação especial.

Os médicos devem estar preparados para orientar as famílias para os serviços públicos que lhes permitam usufruir dos recursos necessários. De notar ainda que, quando os casos suspeitos são referenciados às consultas multidisciplinares de Desenvolvimento, deve entretanto, sem demora, ser excluído o défice auditivo, desencadeados os apoios de intervenção educativa locais, sinalizando a família e a criança para a equipa de intervenção precoce para que seja elaborado um plano individual educativo (que deve estar a cargo das equipas locais de intervenção precoce do Sistema Nacional de Intervenção Precoce na Infância [SNIPI], de acordo com decreto-lei n²81/2009 de 6 de Outubro) e ainda esclarecidos os pais acerca dos défices e problemas da criança e como lidar com eles.

Em conclusão, o autismo é uma perturbação do neurodesenvolvimento crónica, de gravidade variável, que se manifesta essencialmente nos primeiros dois anos de vida. Em regra, apresenta-se como um atraso global 
no desenvolvimento psicomotor, mas é a área da interacção e da comunicação social que está mais envolvida, sendo o seu défice uma especificidade dos quadros de autismo. O diagnóstico deve ser precoce porque as estratégias de intervenção são conhecidas e eficazes.

Os médicos dos cuidados primários devem estar alertados para esta patologia e seguir as estratégias de vigilância do desenvolvimento e do rastreio aos 18 e 24 meses, referenciando atempadamente estas famílias para as consultas de Desenvolvimento.

\section{REFERÊNCIAS BIBLIOGRÁFICAS}

1. Kanner L. Autistic disturbances of affective contact. The Nervous Child 1943; 2: 217-50.

2. Hans Asperger (translated and annotated by Frith U). "Autistic psychopathy" in childhood. In: Frith U, editor. Autism and Asperger Syndrome.Cambridge: Cambridge University Press; 1991. p. 37-92.

3. Rapin I. Classification and causal issues in autism. In: Cohen DJ, Volkmar FR, editors. Handbook of autism and pervasive developmental disorder. 2nd ed. New York: Wiley; 1997. p. 847-67.

4. Volkmar FR, Klin A, Cohen JD. Diagnosis and classification of autism and related conditions: consensus and issues. In: Cohen DJ, Volkmar FR, editors. Handbook of autism and pervasive developmental disorder. 2nd ed. New York: Wiley; 1997. p. 5-40.

5. Rutter M. Diagnostic validity in child psychiatry. Adv Biol Psychiatry 1978; 2: 2-22.

6. Muhle R, Trentacoste SV, Rapin I. The genetics of autism. Pediatrics 2004 May; 113 (5): e472-86

7. American Psychiatric Association. Diagnostic and statistical manual of mental disorders. 3rd ed. Washington, DC: American Psychiatric Association; 1987.

8. American Psychiatric Association. Diagnostic and statistical manual of mental disorders. 4th ed. Washington, DC: American Psychiatric Association; 1994.

9. American Psychiatric Association. Diagnostic and statistical manual of mental disorders. 4th ed., textrevision. Washington, DC: American Psychiatric Association; 2000.

10. World Health Organization. The ICD-10 classification of mental and behavioural disorders: clinical descriptions and diagnostic guideline. Geneva:WHO; 1992.

11. Oliveira G, Ataíde A, Marques C, Miguel TS, Coutinho AM, Mota-Vieira $L$, et al. Epidemiology of autism spectrum disorder in Portugal: prevalence, clinical characterization, and medical conditions. Dev Med Child Neurol 2007 Oct; 49 (10): 726-33.

12. Myers M, Johnson CP;American Academy of Pediatrics Council on Children With Disabilities. Management of children with autism spectrum disorders. Pediatrics 2007 Nov; 120 (5): 1162-82.

13. Committee on Children With Disabilities. Developmental Surveillance and Screening of Infants and Young Children. Pediatrics 2001 Jul; 108 (1): 192-6.

14. Council on Children With Disabilities; Section on Developmental Behavioral Pediatrics; Bright Futures Steering Committee; Medical Home Initiatives for Children With Special Needs Project Advisory Commit- tee. Identifying infants and young children with developmental disorders in the medical home: an algorithm for developmental surveillance and screening. Pediatrics 2006 Jul;118 (1); 405-20.

15. Johnson CP, Myers SM;American Academy of Pediatrics Council on Children With Disabilities. Identification and evaluation of children with autism spectrum disorders. Pediatrics 2007 Nov;120 (5): 1183-215.

16. Bauman ML, Kemper TL. The neuropathology of the autism spectrum disorders: what have we learned? Novartis Found Symp 2003; 112-22.

17. Courchesne $E$, Carper R, Akshoomoff N. Evidence of brain overgrowth in the first year of life in autism. JAMA 2003 Jul 16; 290 (3): 337-44.

18. Osterling JA, Dawson G, Munson JA. Early recognition of 1-year-old infants with autism spectrum disorder versus mental retardation.Dev Psychopathol 2002 Spring; 14 (2): 239-51.

19. Charman T, Swettenham J, Baron-Cohen S, Cox A, Baird G, Drew A. Infants with autism: an investigation of empathy, pretend play, joint attention, and imitation. Dev Psychol 1997 Sep; 33 (5): 781-9.

20. Mundy P, Sullivan L, Mastergeorge AM. A parallel and distributed processing model of joint attention, social cognition and autism. Autism Res 2009 Feb; 2 (1): 2-21.

21. Kleinman M, Robins L, Ventola PE, Pandey J, Boorstein HC, Esser EL, et al. The modified checklist for autism in toddlers: a follow-up study investigating the early detection of autism spectrum disorders.J Autism Dev Disord 2008 May; 38 (5): 827-39.

22. Robbins DL. Modified Checklist for Autism in Toddlers. Disponível em: http://www2.gsu.edu/ psydlr/Diana_L._Robins,_Ph.D._files/MCHAT_new.pdf [acedido a 03/11/2009].

23. Zwaigenbaum L, Bryson S, Lord C, Rogers S, Carter A, Carver L, et al. Clinical assessment and management of toddlers with suspected autism spectrum disorder: insights from studies of high-risk infants. Pediatrics 2009 May; 123 (5): 1383-91.

24. Bryson SE, Zwaigenbaum L, McDermott C, Rombough V, Brian J. The Autism Observation Scale for Infants: scale development and reliability data. J Autism Dev Disord 2008 Apr; 38 (4): 731-8.

25. Stone WL, Lee EB, Ashford L, Brissie J, Hepburn SL, Coonrod EE, et al. Can autism bee diagnosed accurately in children under 3 years?. J Child Psychol Psychiatry 1999 Feb; 40 (2): 219-26.

26. Howlin P, Asgharian A.The diagnosis of autism and Asperger syndrome: findings from a survey of 770 families. Dev Med Child Neurol 1999 Dec; 41 (12): 834-9.

A autora declarou não possuir conflitos de interesses

\section{ENDEREÇO PARA CORRESPONDÊNCIA}

Guiomar Oliveira

Unidade de Neurodesenvolvimento e Autismo

Centro Desenvolvimento Luís Borges, Hospital Pediátrico - Centro

Hospitalar Coimbra, Portugal.

E-mail: guiomar@chc.min-saude.pt 


\section{ABSTRACT}

\section{AUTISM}

Primary Healthcare

Autism is a complex and chronic neurodevelopmental disorder that is common and usually severe. The main goal of this article is to provide guidelines for screening and monitoring of the clinical signs that characterize autism. In most cases, these neurodevelopmental abnormalities are evident before the age of two. Classical clinical signs of autism include difficulties in social relationships and verbal and nonverbal skills as well as a rigid and repetitive behaviour. The author proposes the use of a specific screening test for autism, the Modified Checklist for Autism in Toddlers (M-CHAT), at the ages of 18 and 24 months. Suspected cases should be referred to the specialized multidisciplinary neurodevelopmental teams at Developmental Centers.

Keywords: Autism; Autism Spectrum Disorders; Signs; Screening Test. 